\title{
Lucratividade e risco de sistemas de produção de grãos combinados com pastagens de inverno(1)
}

\author{
Ivo Ambrosi ${ }^{(2)}$, Henrique Pereira dos Santos ${ }^{(2)}$, \\ Renato Serena Fontaneli( ${ }^{(2)}$ e Sandra Maria Zoldan ${ }^{(3)}$
}

\begin{abstract}
Resumo - O objetivo deste trabalho foi avaliar a lucratividade e o risco de quatro sistemas de produção de grãos combinados com pastagens de inverno, em sistema de plantio direto. O experimento foi realizado em Passo Fundo, RS, no período de 1990 a 1995. Os sistemas foram: sistema I (trigo/soja, aveiapreta pastejada/soja, e aveia-preta pastejada/soja); sistema II (trigo/soja e aveia-preta + ervilhaca pastejadas/milho); sistema III (trigo/soja, aveia-preta + ervilhaca pastejadas/soja e aveia-preta + ervilhaca pastejadas/milho); e sistema IV (trigo/soja, aveia-branca/soja e aveia-branca/soja). O delineamento experimental foi em blocos ao acaso, com três repetições, e parcelas totalizando $500 \mathrm{~m}^{2}$. Foram aplicados três tipos de análise à receita líquida dos sistemas: análise da média variância, da distribuição de probabilidade acumulada e da dominância estocástica. Pela análise da média variância, não houve diferença significativa entre os sistemas estudados. Pela distribuição de probabilidade acumulada, a escolha da alternativa depende única e exclusivamente do nível de risco escolhido pelo tomador de decisão. Pelo método da dominância estocástica, o sistema II mostrou ser a melhor alternativa de produção a ser oferecida aos agricultores, dos pontos de vista de rentabilidade e de menor risco.
\end{abstract}

Termos para indexação: fatores de produção, custos, rentabilidade, modelos matemáticos, análise estatística.

\section{Profitability and risk of production systems of grains combined with winter pastures}

Abstract - The objective of this work was to evaluate the profitability and risk of grain production systems combined with annual winter pastures, under no-tillage system. The experiment was carried out in Passo Fundo, RS, Brazil, in a six-year period (1990-1995). The systems were: system I (wheat/ soybean, grazed black oats/soybean, and grazed black oats/soybean); system II (wheat/soybean and grazed black oats + grazed common vetch/corn); system III (wheat/soybean, grazed black oats + grazed common vetch/soybean, and grazed black oats + grazed common vetch/corn); and system IV (wheat/ soybean, white oats/soybean, and white oats/soybean). An experimental design in blocks at random, with three replications and plots with $500 \mathrm{~m}^{2}$, was used. Three types of analysis were applied to the net return of systems: mean-variance analysis, distribution of accumulated probability, and stochastic dominance. When mean-variance analysis was used no significant differences were observed in the systems studied. By the distribution of accumulated probability, the selection of an alternative depends solely and exclusively on the level of risk chosen by the decision-maker. By the stochastic dominance method, system II showed to be the best production alternative to be offered to farmers from both profit and lower risk standpoints.

Index terms: production factors, costs, profitability, mathematical models, statistical analysis.

(1) Aceito para publicação em 28 de novembro de 2000 Trabalho parcialmente realizado com recursos da Fapergs.

(2) Embrapa-Centro Nacional de Pesquisa de Trigo, Caixa Postal 451, CEP 99001-970 Passo Fundo, RS. E-mail ambrosi@cnpt.embrapa.br, hpsantos@cnpt.embrapa.br, renato@cnpt.embrapa.br

(3) Rua João de Cesaro, 29, ap. 304, CEP 99070-140 Passo Fundo, RS

\section{Introdução}

A pecuária é vista, muitas vezes, como uma atividade que dificulta a agricultura, principalmente quando se trata do sistema plantio direto (Dijkstra, 1993). A tecnificação da pecuária como atividade isolada, seja para produção de carne ou de leite, visando a 
atingir produtividade competitiva traz custos fixos elevados. A necessidade de mão-de-obra especializada, tanto no manejo de animais quanto na maquinaria necessária, é um custo que deve ser diluído quando se faz a integração dessas atividades.

No Sul do Brasil, as culturas de melhor retorno econômico são as de verão, e, por mais que se diversifiquem as de inverno, existem poucas possibilidades de renda (Dijkstra, 1993). Com a cultura de trigo, por exemplo, consegue-se obter, em média, pouco mais do que o custo variável, e ocupar, no máximo, 50\% da área de plantio (Dijkstra, 1993).

Com a introdução de risco na teoria da produção, os economistas agrícolas podem fornecer aos agricultores informações econômicas sobre novas tecnologias geradas pela pesquisa, as quais não se referem apenas à rentabilidade de determinada tecnologia, mas também ao risco a que o agricultor estará se expondo na sua adoção (Porto et al., 1982).

Existem vários modelos matemáticos que incorporam risco em decisões individuais e que apresentam forte poder de discriminação entre diferentes alternativas (Cruz, 1986). Entre esses modelos, destacam-se o de média variância, o de distribuição de probabilidade acumulada e o de dominância estocástica (Cruz, 1984).

A análise da média variância (Feldstein, 1969) presume que o tomador de decisão escolha a alternativa que apresente menor variância com relação a uma mesma média ou alternativa que apresente maior média relacionada a um nível igual de variância. A análise da distribuição de probabilidade acumulada (Anderson, 1976) baseia-se no critério da segurança, em primeiro lugar, ou seja, na possibilidade de um dos tratamentos apresentar determinada receita líquida. Nesse caso, os dados são gerados a partir da distribuição completa de probabilidade da distribuição normal dentro de cada tratamento estudado. O próprio sistema de computador divide essa distribuição em 20 intervalos de $5 \%$ de probabilidade em cada tratamento. A análise da dominância estocástica (Hanoch \& Levy, 1970) leva em conta toda a distribuição cumulativa dos retornos de cada tratamento. Esse modelo tem a vantagem de reduzir consideravelmente o número de alternativas eficientes, porque dispõe de alto nível de discriminação.
Na pesquisa conduzida por Santos et al. (1998b) em sistemas de rotação de culturas com cevada, durante dez anos, no sistema de plantio direto, o sistema II (cevada/soja e ervilhaca/milho ou cevada/soja ou aveia-branca/soja) foi separado pela menor variância e pela maior média. Em sistemas de rotação de culturas com trigo, durante dez anos, no sistema de plantio direto, o sistema II (trigo/soja e ervilhaca/milho ou trigo/soja e aveia-branca/soja) destacou-se como o mais lucrativo e de menor risco (Santos et al., 2000).

Este trabalho teve por objetivo avaliar a lucratividade e o risco de sistemas de produção de grãos combinados com pastagens anuais de inverno.

\section{Material e Métodos}

Os dados de dois ciclos de rotação de culturas usados neste trabalho foram obtidos de um experimento de sistemas de produção de grãos combinados com pastagens anuais de inverno, instalado no Centro de Extensão e Pesquisa Agronômica, da Faculdade de Agronomia e Medicina Veterinária da Universidade de Passo Fundo, em Passo Fundo, RS, de 1990 a 1995, em solo classificado como Latossolo Vermelho-Escuro distrófico (Brasil, 1973).

As análises de risco foram determinadas no experimento de produção de grãos combinados com pastagens anuais de inverno, composto de quatro sistemas: sistema I (trigo/ soja, aveia-preta pastejada/soja e aveia-preta pastejada/ soja), sistema II (trigo/soja e aveia-preta + ervilhaca pastejadas/milho), sistema III (trigo/soja, aveia-preta + ervilhaca pastejadas/soja e aveia-preta + ervilhaca pastejadas/milho) e sistema IV (trigo/soja, aveia-branca/ soja e aveia-branca/soja) (Tabela 1). Em 1990 havia trevo vesiculoso no lugar de ervilhaca. As culturas, tanto no inverno como no verão, foram estabelecidas sob sistema plantio direto.

A adubação de manutenção para cada cultura foi realizada com base na análise de solo. As amostras de solo foram coletadas após cada rotação de três anos, depois das culturas de verão.

As épocas de semeadura, o controle de plantas daninhas e os tratamentos fitossanitários foram realizados de acordo com a recomendação para cada cultura, e a colheita foi realizada com automotriz especial para parcelas. O rendimento de grãos (aveia-branca, milho, soja e trigo) foi determinado a partir da colheita de $1 / 3$ da parcela, ajustando-se para umidade de $13 \%$.

O pastejo de aveia-preta e de ervilhaca foi realizado por bovinos mistos (corte e leite), com 15 a 18 animais 
por parcela, quando a aveia-preta atingiu estatura de, aproximadamente, $30 \mathrm{~cm}$, deixando-se uma altura de resteva de $7 \mathrm{~cm}$ a $10 \mathrm{~cm}$. Os bovinos eram colocados nas parcelas quando o solo não apresentava excesso de umidade e geralmente consumiam a forragem disponível no primeiro dia. Realizaram-se dois a três pastejos por ano, geralmente em junho, julho e agosto. Foram avaliados o peso de matéria verde, antes e depois do pastejo, e, posteriormente, o de matéria seca. Após o último pastejo, permitiu-se um rebrote entre 30 e 40 dias, até acumular uma cobertura verde de 1,5 a $2,0 \mathrm{t}$ de matéria seca por hectare, para, então, fazer a dessecação e semear as culturas de verão.

$\mathrm{O}$ peso animal foi estimado por meio do consumo de matéria seca. A conversão considerada foi de $10 \mathrm{~kg}$ de forragem seca consumida para $1 \mathrm{~kg}$ de ganho de peso vivo dos animais (Moojen \& Saibro, 1981; Moraes, 1991).

Entende-se por receita líquida a diferença entre a receita bruta (rendimento de grãos das espécies em estudo e ganho de peso animal $\mathrm{x}$ preço de venda como produto comercial) e os custos totais [custos variáveis (custos dos insumos + custos das operações de campo) e custos fixos (exemplo: depreciação linear de máquinas e equipamentos e juros de $6 \%$ sobre o capital)]. Os custos médios com insumos, com operações de campo e com a venda de produtos foram obtidos em dezembro de 1995, na região de Passo Fundo (Tabela 2). $\mathrm{O}$ uso de preços pontuais tem o objetivo de eliminar os problemas causados pela inflação, quando se analisam resultados de séries temporais.

O delineamento experimental foi o de blocos ao acaso, com três repetições. A área de cada parcela foi de $500 \mathrm{~m}^{2}$. Foi efetuada a análise de média variância da receita líqui- da da média dos anos (1990 a 1995). As médias foram comparadas entre si pelo teste de Duncan, a 5\% de probabilidade.

Paralelamente, foi aplicado à receita líquida o programa para computador "Biorisco" ou "Pacta", que é baseado no critério de simetria de Hanoch \& Levy (1970). Esse programa compara as alternativas, duas a duas, dos pontos de vista de rentabilidade e de risco (distribuição de probabilidade acumulada, "twentiles", e dominância estocástica de 2 o grau, "pairwise"), conforme Cruz (1980).

\section{Resultados e Discussão}

Os rendimentos de grãos de cada espécie e o ganho de peso dos animais, de 1990 a 1995, nos diferentes sistemas de produção de grãos com pastagens anuais de inverno, encontram-se na Tabela 3. A receita líquida média conjunta por hectare referente aos quatro sistemas de produção, a distribuição de probabilidade acumulada da receita líquida (twentiles) dos sistemas e os resultados da análise da dominância estocástica (pairwise) dos sistemas, em comparação à receita líquida, podem ser verificados nas Tabelas 4 a 6.

A receita líquida do sistema I $(\mathrm{R} \$ 377,93)$ não diferiu significativamente, pela análise da média variância, dos sistemas II ( $\$$ \$ 432,71), III (R\$ 400,27) e IV (R \$ 322,93) (Tabela 4). Assim, a simples análise da receita líquida através da média variância não permitiu separar, entre os sistemas estu-

Tabela 1. Sistemas de produção de grãos combinados com pastagens anuais de inverno, em sistema plantio direto. Passo Fundo, $\mathrm{RS}^{(1)}$.

\begin{tabular}{|c|c|c|c|c|c|c|}
\hline \multirow{2}{*}{$\begin{array}{l}\text { Sistema } \\
\text { de produção }\end{array}$} & \multicolumn{6}{|c|}{ Ano } \\
\hline & 1990 & 1991 & 1992 & 1993 & 1994 & 1995 \\
\hline \multirow[t]{3}{*}{ Sistema I } & $\mathrm{T} / \mathrm{S}$ & $\mathrm{Ap} / \mathrm{S}$ & $\mathrm{Ap} / \mathrm{S}$ & $T / S$ & $\mathrm{Ap} / \mathrm{S}$ & $\mathrm{Ap} / \mathrm{S}$ \\
\hline & $\mathrm{Ap} / \mathrm{S}$ & $\mathrm{Ap} / \mathrm{S}$ & $\mathrm{T} / \mathrm{S}$ & $\mathrm{Ap} / \mathrm{S}$ & $\mathrm{Ap} / \mathrm{S}$ & $\mathrm{T} / \mathrm{S}$ \\
\hline & $\mathrm{Ap} / \mathrm{S}$ & $T / S$ & $\mathrm{Ap} / \mathrm{S}$ & $\mathrm{Ap} / \mathrm{S}$ & $T / S$ & $\mathrm{Ap} / \mathrm{S}$ \\
\hline \multirow[t]{2}{*}{ Sistema II } & $T / S$ & $\mathrm{Ap}+\mathrm{E} / \mathrm{M}$ & $T / S$ & $\mathrm{Ap}+\mathrm{E} / \mathrm{M}$ & $T / S$ & $\mathrm{Ap}+\mathrm{E} / \mathrm{M}$ \\
\hline & $A p+T v / M$ & $T / S$ & $\mathrm{Ap}+\mathrm{E} / \mathrm{M}$ & $T / S$ & $\mathrm{Ap}+\mathrm{E} / \mathrm{M}$ & $\mathrm{T} / \mathrm{S}$ \\
\hline \multirow[t]{3}{*}{ Sistema III } & $T / S$ & $A p+E / S$ & $\mathrm{Ap}+\mathrm{E} / \mathrm{M}$ & $T / S$ & $A p+E / S$ & $\mathrm{Ap}+\mathrm{E} / \mathrm{M}$ \\
\hline & $\mathrm{Ap}+\mathrm{Tv} / \mathrm{S}$ & $\mathrm{Ap}+\mathrm{E} / \mathrm{M}$ & $\mathrm{T} / \mathrm{S}$ & $\mathrm{Ap}+\mathrm{E} / \mathrm{S}$ & $\mathrm{Ap}+\mathrm{E} / \mathrm{M}$ & $\mathrm{T} / \mathrm{S}$ \\
\hline & $\mathrm{Ap}+\mathrm{Tv} / \mathrm{M}$ & $\mathrm{T} / \mathrm{S}$ & $\mathrm{Ap}+\mathrm{E} / \mathrm{S}$ & $\mathrm{Ap}+\mathrm{E} / \mathrm{M}$ & $\mathrm{T} / \mathrm{S}$ & $\mathrm{Ap}+\mathrm{E} / \mathrm{S}$ \\
\hline \multirow[t]{3}{*}{ Sistema IV } & $\mathrm{T} / \mathrm{S}$ & $\mathrm{Ab} / \mathrm{S}$ & $\mathrm{Ab} / \mathrm{S}$ & $\mathrm{T} / \mathrm{S}$ & $\mathrm{Ab} / \mathrm{S}$ & $\mathrm{Ab} / \mathrm{S}$ \\
\hline & $\mathrm{Ab} / \mathrm{S}$ & $\mathrm{Ab} / \mathrm{S}$ & $T / S$ & $\mathrm{Ab} / \mathrm{S}$ & $\mathrm{Ab} / \mathrm{S}$ & $\mathrm{T} / \mathrm{S}$ \\
\hline & $\mathrm{Ab} / \mathrm{S}$ & $T / S$ & $\mathrm{Ab} / \mathrm{S}$ & $\mathrm{Ab} / \mathrm{S}$ & $T / S$ & $T / S$ \\
\hline
\end{tabular}

${ }^{(1)} \mathrm{Ab}$ : aveia-branca; Ap: aveia-preta; E: ervilhaca; M: milho; S: soja; T: trigo; Tv: trevo vesiculoso. 
Tabela 2. Preço unitário (R\$) de venda dos produtos e dos insumos usados, por quilograma, por tonelada ou por litro, em dezembro de 1995. Passo Fundo, RS.

\begin{tabular}{|c|c|}
\hline Produto/insumo & Preço $(\mathrm{R} \$)$ \\
\hline \multicolumn{2}{|l|}{ Produtos } \\
\hline Aveia-preta- carne & $700,00 \mathrm{t}^{-1}$ \\
\hline Aveia-branca & $130,00 \mathrm{t}^{-1}$ \\
\hline Aveia-preta+ ervilhaca-carne & $700,00 \mathrm{t}^{-1}$ \\
\hline Milho & $110,00 \mathrm{t}^{-1}$ \\
\hline Soja & $245,00 \mathrm{t}^{-1}$ \\
\hline Trigo & $180,00 \mathrm{t}^{-1}$ \\
\hline \multicolumn{2}{|l|}{ Fertilizantes } \\
\hline $\mathrm{N}$ & $833,00 \mathrm{t}^{-1}$ \\
\hline $\mathrm{P}_{2} \mathrm{O}_{5}$ & $700,00 \mathrm{t}^{-1}$ \\
\hline $\mathrm{K}_{2} \mathrm{O}$ & $416,00 \mathrm{t}^{-1}$ \\
\hline Calcário & $30,30 \mathrm{t}^{-1}$ \\
\hline \multicolumn{2}{|l|}{ Fungicidas } \\
\hline Iprodione + thiran & $2,60 \mathrm{~kg}^{-1}$ \\
\hline Propiconazole & $43,00 \mathrm{~L}^{-1}$ \\
\hline Triadimenol & $5,00 \mathrm{~kg}^{-1}$ \\
\hline \multicolumn{2}{|l|}{ Herbicidas } \\
\hline $2,4 \mathrm{D}$ & $6,35 \mathrm{~L}^{-1}$ \\
\hline Atrazine + metolachlor & $6,10 \mathrm{~L}^{-1}$ \\
\hline Atrazine + simazine & $5,45 \mathrm{~L}^{-1}$ \\
\hline Clomazobe & $37,00 \mathrm{~L}^{-1}$ \\
\hline Diclofob-methyl & $17,76 \mathrm{~L}^{-1}$ \\
\hline Diuron & $6,35 \mathrm{~L}^{-1}$ \\
\hline Diuron + paraquat & $9,20 \mathrm{~L}^{-1}$ \\
\hline Glifosate & $7,87 \mathrm{~L}^{-1}$ \\
\hline Imazaquin & $30,46 \mathrm{~L}^{-1}$ \\
\hline Metribuzin & $23,50 \mathrm{~L}^{-1}$ \\
\hline Paraquat & $9,27 \mathrm{~L}^{-1}$ \\
\hline Trifluralina & $7,40 \mathrm{~L}^{-1}$ \\
\hline \multicolumn{2}{|l|}{ Inseticidas } \\
\hline Formicida & $4,50 \mathrm{~kg}^{-1}$ \\
\hline Lambdacioletrin & $30,00 \mathrm{~L}^{-1}$ \\
\hline Monocrotofós & $9,00 \mathrm{~L}^{-1}$ \\
\hline Permitrina & $52,00 \mathrm{~L}^{-1}$ \\
\hline Triclorfon & $7,73 \mathrm{~L}^{-1}$ \\
\hline
\end{tabular}

dados, a melhor alternativa a ser oferecida aos agricultores. Na primeira avaliação deste experimento (1990 a 1992), Ambrosi \& Fontaneli (1994) e Santos et al. (1998a), trabalhando com sistemas de rotação de culturas com triticale, obtiveram resultados similares.

A análise da média variância (Feldstein, 1969) nem sempre possibilita a escolha da melhor alternativa. Nesse caso, pode ser usado o critério da distribuição de probabilidade acumulada da receita líquida (Anderson, 1976). Esse tipo de análise possibilita a escolha da alternativa com base numa determinada probabilidade de garantir renda líquida, em dado nível de escolha do tomador de decisão. Esse princípio baseia-se no critério da segurança em primeiro lugar, ou seja, na possibilidade de um dos sistemas apresentar renda líquida de $0 \%$ a $100 \%$ de probabilidade. $\mathrm{O}$ valor seria escolhido pelo tomador de decisão.

Pela análise da distribuição de probabilidade acumulada da receita líquida (Tabela 5), o sistema I apresentou, na baixa probabilidade de risco (5\%), maior renda líquida/ha (R\$19,29), em comparação com os sistemas II (R\$ 0,00), III (R\$ 0,00$)$ e IV $(\mathrm{R} \$ 0,00)$. $\mathrm{Na}$ alta probabilidade de risco (100\%), o sistema II obteve a maior renda líquida/ha ( $\mathrm{R} \$ 1.380,56)$, em relação aos sistemas I (R\$1.030,89), III (R\$ $1.229,61)$ e IV (R\$ 923,10). Na primeira avaliação (1990 a 1992) deste experimento (Ambrosi $\&$ Fontaneli, 1994) e no trabalho de Santos et al. (1998b) com sistemas de rotação de culturas com cevada, os autores não conseguiram separar o mesmo sistema no baixo ou no alto nível de probabilidade de risco.

Supondo-se que um agricultor " $A$ " não queira correr risco superior a 5\% de ter receita líquida negativa, esse agricultor jamais deverá escolher os sistemas II, III ou IV (Tabela 5). Por outro lado, um agricultor "B", que pretenda obter a maior renda líquida possível, escolheria o sistema II. Um agricultor "C", que jogasse $50 \%$ de suas possibilidades de atingir a máxima receita líquida também escolheria o sistema II para obter uma receita líquida menor ou igual a R \$ 423,96/ha. Para esse método, a escolha da alternativa depende única e exclusivamente do nível de risco escolhido pelo tomador de decisão.

Pela dominância estocástica de $2^{\circ}$ grau, o sistema II dominou os demais sistemas estudados (Tabela 6). Por sua vez, o sistema III dominou os sistemas I e IV, e o sistema I dominou o sistema IV. Os sistemas podem ser classificados na seguinte ordem decrescente: sistema II, sistema III e sistema I, sendo o sistema IV o pior em termos de rentabilidade e de risco.

O método de análise através da dominância estocástica apresentou maior nível de discriminação do que o método da média variância e deve ser empregado sempre que possível, para testar as novas recomendações aos agricultores. Segundo Porto et al. (1982), esse método oferece opções dentro de um leque limitado. Neste experimento, de quatro sistemas, somente um foi separado. 
Tabela 3. Rendimento de grãos de espécies e ganho de peso de animais com pastagens de inverno de quatro sistemas de produção. Passo Fundo, RS ${ }^{(1)}$.

\begin{tabular}{|c|c|c|c|c|c|c|c|c|c|c|c|}
\hline \multicolumn{12}{|c|}{ Ano } \\
\hline \multicolumn{2}{|c|}{1990} & \multicolumn{2}{|c|}{1991} & \multicolumn{2}{|c|}{1992} & \multicolumn{2}{|c|}{1993} & \multicolumn{2}{|c|}{1994} & \multicolumn{2}{|c|}{1995} \\
\hline \multicolumn{12}{|c|}{ Sistema I } \\
\hline $\mathrm{T}$ & S & Ap & S & Ap & $S$ & $\mathrm{~T}$ & S & Ap & S & Ap & S \\
\hline 1.100 & 1.550 & $207^{(2)}$ & 2.508 & $374^{(2)}$ & 2.336 & 1.870 & 2.667 & $345^{(2)}$ & 2.820 & $266^{(2)}$ & 3.220 \\
\hline Ap & S & Ap & $\mathrm{S}$ & $\mathrm{T}$ & $\mathrm{S}$ & Ap & $\mathrm{S}$ & Ap & S & $\mathrm{T}$ & $\mathrm{S}$ \\
\hline $263^{(2)}$ & 800 & $279^{(2)}$ & 2.650 & 2.980 & 2.845 & $229^{(2)}$ & 2.567 & $300^{(2)}$ & 2.775 & 2.608 & 2.860 \\
\hline Ap & $\mathrm{S}$ & $\mathrm{T}$ & $\mathrm{S}$ & Ap & $S$ & Ap & $\mathrm{S}$ & $\mathrm{T}$ & $\mathrm{S}$ & Ap & $\mathrm{S}$ \\
\hline $305^{(2)}$ & 900 & 2.967 & 2.558 & $412^{(2)}$ & 2.068 & $278^{(2)}$ & 2.675 & 2.560 & 2.668 & $255^{(2)}$ & 3.214 \\
\hline \multicolumn{12}{|c|}{ Sistema II } \\
\hline $\mathrm{T}$ & S & $\mathrm{Ap}+\mathrm{E}$ & M & $\mathrm{T}$ & S & $A p+E$ & M & $\mathrm{T}$ & S & $A p+E$ & M \\
\hline 1.233 & 1.650 & $229^{(2)}$ & 9.683 & 2.534 & 2.568 & $247^{(2)}$ & 7.408 & 2.153 & 2.747 & $286^{(2)}$ & 5.102 \\
\hline $\mathrm{Ap}+\mathrm{Tv}$ & M & $\mathrm{T}$ & $\mathrm{S}$ & $A p+E$ & M & $\mathrm{T}$ & S & $A p+E$ & M & $\mathrm{T}$ & $\mathrm{S}$ \\
\hline $277^{(2)}$ & 1.083 & 2.317 & 2.891 & $375^{(2)}$ & 8.916 & 1.293 & 2.767 & $342^{(2)}$ & 8.445 & 2.384 & 3.402 \\
\hline \multicolumn{12}{|c|}{ Sistema III } \\
\hline $\mathrm{T}$ & S & $A p+E$ & S & $A p+E$ & M & $\mathrm{T}$ & S & $A p+E$ & S & $A p+E$ & M \\
\hline 1.158 & 1.733 & $242^{(2)}$ & 2.600 & $438^{(2)}$ & 8412 & 1.661 & 2.691 & $355^{(2)}$ & 2.861 & $327^{(2)}$ & 5.491 \\
\hline $\mathrm{Ap}+\mathrm{Tv}$ & $\mathrm{S}$ & $A p+E$ & M & $\mathrm{T}$ & $S$ & $A p+E$ & $\mathrm{~S}$ & $A p+E$ & M & $\mathrm{T}$ & $\mathrm{S}$ \\
\hline $325^{(2)}$ & 950 & $240^{(2)}$ & 8.483 & 2.672 & 3.022 & $299^{(2)}$ & 2.833 & $358^{(2)}$ & 7.247 & 2.484 & 3.158 \\
\hline $\mathrm{Ap}+\mathrm{Tv}$ & M & $\mathrm{T}$ & $\mathrm{S}$ & $A p+E$ & $\mathrm{~S}$ & $A p+E$ & M & $\mathrm{T}$ & $\mathrm{S}$ & $A p+E$ & $\mathrm{~S}$ \\
\hline $274^{(2)}$ & 755 & 2.733 & 3.008 & $442^{(2)}$ & 2.445 & $265^{(2)}$ & 5.458 & 2.404 & 2.845 & $316^{(2)}$ & 3.278 \\
\hline \multicolumn{12}{|c|}{ Sistema IV } \\
\hline $\mathrm{T}$ & $S$ & $\mathrm{Ab}$ & S & $\mathrm{Ab}$ & S & $\mathrm{T}$ & S & $\mathrm{Ab}$ & S & $\mathrm{Ab}$ & S \\
\hline 1.067 & 1.383 & 1.800 & 2.275 & 2.089 & 2.092 & 1.551 & 2.542 & 683 & 2.606 & 1.730 & 3.334 \\
\hline $\mathrm{Ab}$ & S & $\mathrm{Ab}$ & $\mathrm{S}$ & $\mathrm{T}$ & $S$ & $\mathrm{Ab}$ & $S$ & $\mathrm{Ab}$ & $S$ & $\mathrm{~T}$ & $S$ \\
\hline 2.350 & 1.450 & 1.733 & 2.458 & 2.560 & 1.916 & 1.784 & 2.758 & 597 & 2.887 & 2.355 & 3.299 \\
\hline $\mathrm{Ab}$ & $\mathrm{S}$ & $\mathrm{T}$ & $\mathrm{S}$ & $\mathrm{Ab}$ & $S$ & $\mathrm{Ab}$ & $\mathrm{S}$ & $\mathrm{T}$ & $\mathrm{S}$ & $\mathrm{Ab}$ & $\mathrm{S}$ \\
\hline 2.300 & 1.317 & 2.733 & 1.992 & 2.390 & 1.856 & 1.612 & 2.575 & 2.329 & 2.744 & 1.638 & 3.426 \\
\hline
\end{tabular}

(1) Ab: aveia-branca; Ap: aveia-preta; E: ervilhaca; M: milho; S: soja; T: trigo. ${ }^{(2)}$ Ganho de peso de animais.

Tabela 4. Receita líquida média anual ( $\mathrm{R} \$ / \mathrm{ha})$, em sistemas de produção de grãos combinados com pastagens anuais de inverno. Passo Fundo, $\mathrm{RS}^{(1)}$.

\begin{tabular}{lcc}
\hline $\begin{array}{l}\text { Sistema de } \\
\text { produção }\end{array}$ & $\begin{array}{c}\text { Receita líquida média } \\
1990 \text { a } 1995\end{array}$ & Desvio padrão \\
\hline Sistema I & $377,93^{\text {ns }}$ & 229,93 \\
Sistema II & 432,71 & 333,77 \\
Sistema III & 400,27 & 292,03 \\
Sistema IV & 322,93 & 211,34 \\
\hline
\end{tabular}

(1)Sistema I: trigo/soja, aveia-preta/soja e aveia-preta/soja; sistema II: trigo/ soja e aveia-preta + ervilhaca/milho; sistema III: trigo/soja, aveia-preta + ervilhaca/soja e aveia-preta + ervilhaca/milho; sistema IV: trigo/soja, aveia-

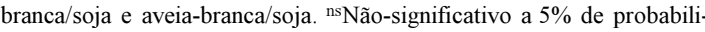
dade, pelo teste de Duncan

O sistema II mostrou-se como a alternativa de menor risco, caso adotado pelos agricultores. De acordo com Moutinho et al. (1978), o risco tende a atuar como impedimento por parte dos agricultores à adoção de novas práticas agrícolas. O sistema II (trigo/soja e aveia-preta + ervilhaca pastejadas/milho) foi o mais lucrativo e seguro, do ponto de vista de risco. Além disso, pela análise econômica, entre os sistemas mistos, o sistema II pode ser considerado uma alternativa confiável para rotacionar com o sistema de grãos (IV) (Fontaneli et al., 2000).

A cultura de milho produziu rendimento de grãos relativamente elevado de 1991 a 1995 , variando de $5.102 \mathrm{~kg} /$ ha a $9.683 \mathrm{~kg} / \mathrm{ha}$ (Tabela 2). Por outro lado, a cultura de soja produziu baixo rendimento de grãos em 1990 (de $800 \mathrm{~kg} / \mathrm{ha}$ a $1.733 \mathrm{~kg} / \mathrm{ha})$, médio, em 1992 e 1993 (de $1.845 \mathrm{~kg} / \mathrm{ha}$ a $3.022 \mathrm{~kg} / \mathrm{ha}$ ), e alto, de 1993 a 1995 (de $2.542 \mathrm{~kg} / \mathrm{ha}$ a $3.426 \mathrm{~kg} / \mathrm{ha}$ ). Isso, por si só, explica o baixo desempenho econômico dos sistemas I e IV, e separou o sistema II do sistema III. A cultura de trigo, que fez parte de todos os sistemas, produziu relativamente bem na maioria dos anos estudados.

Na avaliação do primeiro ciclo de rotação de culturas realizada (Ambrosi \& Fontaneli, 1994), o sistema separado pelo método da dominância estocástica foi o III. Nesta avaliação e nos trabalhos de Santos et al. (1998b, 2000), em sistemas de rotação de culturas com cevada e com trigo, os melhores sistemas foram aqueles com apenas um inverno ou verão de rotação. Deve ser levado em conta que, nos últimos 
Tabela 5. Distribuição de probabilidade acumulada da receita ( $\mathrm{R} \%$ /ha) líquida (twentiles), em sistemas de produção de grãos combinados com pastagens anuais de inverno, de 1990 a 1995. Passo Fundo, RS.

\begin{tabular}{crrrr}
\hline Probabilidade & \multicolumn{4}{c}{ Sistema de produção ${ }^{(1)}$} \\
\cline { 2 - 5 } de risco $(\%)$ & \multicolumn{1}{c}{ I } & \multicolumn{1}{c}{ II } & \multicolumn{1}{c}{ III } & \multicolumn{1}{c}{ IV } \\
\hline 0 & $<0,00$ & $<0,00$ & $<0,00$ & $<0,00$ \\
5 & 19,29 & $<0,00$ & $<0,00$ & $<0,00$ \\
10 & 67,66 & $<0,00$ & 7,42 & 37,75 \\
15 & 100,27 & 29,66 & 47,61 & 67,72 \\
20 & 189,69 & 159,46 & 161,18 & 149,91 \\
25 & 227,06 & 213,71 & 208,65 & 184,26 \\
30 & 250,81 & 248,18 & 238,81 & 206,09 \\
35 & 285,58 & 298,65 & 282,97 & 238,04 \\
40 & 310,81 & 335,27 & 315,02 & 261,23 \\
45 & 343,84 & 383,23 & 356,97 & 291,60 \\
50 & 371,90 & 423,96 & 392,61 & 317,39 \\
55 & 414,49 & 485,78 & 446,70 & 356,53 \\
60 & 465,34 & 559,60 & 511,30 & 403,28 \\
65 & 481,81 & 583,51 & 532,21 & 418,41 \\
70 & 505,07 & 617,26 & 561,75 & 439,79 \\
75 & 539,60 & 667,39 & 605,61 & 471,53 \\
80 & 585,00 & 733,29 & 663,27 & 513,25 \\
85 & 621,53 & 786,33 & 709,67 & 546,84 \\
90 & 673,52 & 861,80 & 775,71 & 594,62 \\
95 & 755,32 & 980,53 & 879,60 & 669,80 \\
100 & $1.030,89$ & $1.380,56$ & $1.229,61$ & 923,10 \\
\hline
\end{tabular}

${ }^{(1)}$ Sistema I: trigo/soja, aveia-preta/soja e aveia-preta/soja; sistema II: trigo/ soja e aveia-preta + ervilhaca/milho; sistema III: trigo/soja, aveia-preta + ervilhaca/soja e aveia-preta +ervilhaca/milho; sistema IV: trigo/soja, aveiabranca/soja e aveia-branca/soja.

Tabela 6. Dominância estocástica da receita líquida dos sistemas de produção de grãos combinados com pastagens de inverno, 1990 a 1995. Passo Fundo, $\mathrm{RS}^{(1)}$.

\begin{tabular}{lcccc}
\hline Sistema de & \multicolumn{4}{c}{ Sistema de produção } \\
\cline { 2 - 5 } produção & I & II & III & IV \\
\hline Sistema I & - & 0 & 0 & 1 \\
Sistema II & 1 & - & 1 & 1 \\
Sistema III & 1 & 0 & - & 1 \\
Sistema IV & 0 & 0 & 0 & - \\
\hline
\end{tabular}

${ }^{(1)}$ Sistema I: trigo/soja, aveia-preta/soja e aveia-preta/soja; sistema II: trigo/ soja e aveia-preta + ervilhaca/milho; sistema III: trigo/soja, aveia-preta + ervilhaca/soja e aveia-preta + ervilhaca/milho; sistema IV: trigo/soja, aveiabranca/soja e aveia-branca/soja; a leitura deve ser feita no sentido horizontal; 0 (zero) significa que a tecnologia da linha é dominada pela da coluna e 1 (um) significa que a tecnologia da linha domina a da coluna.

dois sistemas, estudaram-se somente rendimentos de lavoura, enquanto no referido trabalho constam rendimentos de lavoura associada à pecuária.

O sistema misto (lavoura + pecuária) com um inverno de pastagem e com um de lavoura (sistema II) foi superior aos demais sistemas mistos (sistemas I e III), com dois invernos de pastagens e com um de lavoura, e ao sistema com somente lavoura por três invernos (sistema IV). No verão, em todos os sistemas semeou-se milho ou soja. Dessa forma, ficou claro que a lavoura (sistema de produção) pode ser explorada juntamente com a pecuária (pastagens consorciada, no inverno, para engorda de animais) para aumentar a rentabilidade da propriedade agrícola como um todo.

A engorda de animais durante o período de inverno foi uma alternativa positiva para rotacionar com a lavoura de trigo. Nesse caso, as atividades da propriedade completaram-se sem competir entre si. Segundo Mello (1996), isso já vem ocorrendo no Planalto Médio e nas Missões do Estado do Rio Grande do Sul, desde a década de setenta, com a introdução de novas espécies forrageiras, tanto para a terminação de bovinos de corte como para a alimentação de bovinos leiteiros.

\section{Conclusões}

1. Pela análise da dominância estocástica o sistema II é a melhor alternativa de produção para a região de Passo Fundo, RS, sob o ponto de vista de rentabilidade e de menor risco.

2. Dos três métodos avaliados, o método de dominância estocástica é o mais apropriado para avaliar experimentos com análises econômicas.

3. A integração lavoura-pecuária sob sistema plantio direto é viável para engordar os animais no período invernal e na rotação com culturas de inverno e de verão.

\section{Referências}

AMBROSI, I.; FONTANELI, R. S. Análise de risco de quatro sistemas alternativos de produção de integração lavoura/pecuária. Teoria e Evidência Econômica, Passo Fundo, v. 2, n. 3, p. 129-148, 1994.

ANDERSON, J. R. Modeling decision-making under risk. México: Cimmyt, 1976. Não paginado.

BRASIL. Ministério da Agricultura. Departamento Nacional de Pesquisa Agropecuária. Divisão de Pesquisa Pedológica. Levantamento de reconhecimento dos solos do Estado do Rio Grande do Sul. Recife, 1973. 421 p. (Boletim Técnico, 30).

CRUZ, E. M. da. Aspectos teóricos sobre incorporação de risco em modelos de decisão. In: CRUZ, E. M. da (Ed.). 
Risco em modelos de decisão na agricultura. Brasília: Embrapa-DEP, 1984. p. 13-33. (Embrapa-DEP. Documentos, 15).

CRUZ, E. M. da. Aspectos teóricos sobre incorporação de risco em modelos de decisão. In: CONTINI, E.; ARAÚJO, J. D. de; OLIVEIRA, A. J. de; GARRIDO, W. E. Planejamento da propriedade agrícola: modelos de decisão. 2. ed. rev. Brasília: Embrapa-DDT, 1986. p. 237-260. (Embrapa-DEP. Documentos, 7).

CRUZ, F. R. da. PACTA - Programa de Avaliação Comparativa de Tecnologias Alternativas: guia do usuário, versão 2. Brasília: Embrapa-DDM, 1980. 7 p.

DIJKSTRA, F. A integração agricultura-pecuária no plantio direto. Informações Agronômicas, Piracicaba, n. 63, p. 1-2, set. 1993.

FELDSTEIN, M. S. Mean variance analysis in the theory of liquidity preference and portfolio selection. Review of Economic Studies, Oxford, v. 36, n. 1, p. 5-14, 1969.

FONTANELI, R. S.; AMBROSI, I.; SANTOS, H. P. dos; IGNACZAK, J. C.; ZOLDAN, S. M. Análise econômica de sistemas de produção de grãos com pastagens anuais de inverno, em sistema plantio direto. Pesquisa Agropecuária Brasileira, Brasília, v. 35, n. 11, p. 21292137, nov. 2000

HANOCH, G.; LEVY, H. Efficient portfolio selection with quadratic and cubic utility. Journal of Business, Chicago, v. 43, n. 2, p. 181-189, 1970.

MELLO, J. da S. Fundamentos para integração lavourapecuária no sistema plantio direto. Plantio Direto, Passo Fundo, n. 36, p. 12-13, nov./dez. 1996.

MOOJEN, E. L.; SAIBRO, J. C. de. Efeito de regimes de corte sobre o rendimento e qualidade de misturas forrageiras de estação fria. Pesquisa Agropecuária Brasileira, Brasília, v. 16, n. 1, p. 101-109, jan. 1981.

MORAES, A. de. Produtividade animal e dinâmica de uma pastagem de pangola (Digitaria decumbens), azevém (Lolium multiflorum) e trevo branco (Trifolium repens), submetida a diferentes pressões de pastejo. Porto Alegre: UFRGS, 1991. 200 p. Tese de Doutorado.

MOUTINHO, D. V.; SANDERS JUNIOR, J. H.; WEBER, M. T. Tomada de decisão sob condições de risco em relação à nova tecnologia para a produção de feijão de corda. Revista de Economia Rural, Brasília, v. 16, n. 4, p. 4158, out./dez. 1978.

PORTO, V. H. da F.; CRUZ, E. R. da; INFELD, J. A. Metodologia para incorporação de risco em modelos de decisão usados na análise comparativa entre alternativas: o caso da cultura do arroz irrigado. Revista de Economia Rural, Brasília, v. 20, n. 2, p. 193-211, abr.jun. 1982.

SANTOS, H. P. dos; AMBROSI, I.; LHAMBY, J. C. B.; BAIER, A. C. Análise de risco de sistemas de rotação de culturas com triticale, sob sistema plantio direto. Pesquisa Agropecuária Brasileira, Brasília, v. 33, n. 4, p. 375383, abr. 1998a.

SANTOS, H. P. dos; AMBROSI, I.; SANDINI, I. Análise de risco de sistemas de rotação de culturas com cevada em plantio direto, num período de dez anos. Pesquisa Agropecuária Brasileira, Brasília, v. 33, n. 8, p. 12211227, ago. 1998b.

SANTOS, H. P. dos; AMBROSI, I.; WOBETO, C. Risco de sistemas de rotação de culturas de inverno e verão sob plantio direto. Ciência Rural, Santa Maria, v. 30, n. 1, p. $37-42,2000$. 